\title{
Uso de piedra ignimbrita como sustituto de agregado fino en morteros de cal para restauración
}

Donají Pérez Díaz

donaji.perez.d@gmail.com

Alumna de Posgrado en Ciencias de la Contruccion Facultad de Arquitectura - UABJO Oaxaca, México.

Heidy Gómez Barranco arq.heidy.gb@gmail.com

Profesora de Posgrado en Ciencias de la Construcción Facultad de Arquitectura - UABJO Oaxaca, México.

Herwing López Calvo

herwingzeth@gmail.com

Profesor de Posgrado en Ciencias de la Construcción Facultad de Arquitectura - UABJO Oaxaca, México.

Elia Mercedes Alonso Guzmán

elia.alonso@umich.mx

Profesora Departamento de Materiales

Facultad de Ingeniería Civil - UMSNH Michoacán, México

\section{RESUMEN}

Esta investigación evalúa el uso de la ignimbrita como residuo reciclado de labra con actividad puzolánica en la elaboración de morteros de cal para restauración arquitectónica. Se estudió el efecto de la resistencia a la compresión de morteros de cal donde el agregado principal fue reemplazado por el residuo reciclado y una parte del aglutinante tradicional es sustituido por el polvo de piedra y posteriormente se compararon contra morteros tradicionales. Se utilizaron dos metodologías, una abarca la caracterización de los materiales involucrados en la elaboración de los morteros y otra se refiere a la caracterización de los morteros elaborados durante la campaña experimental. Se utilizó el método cuantitativo-descriptivo, el cual permite responder preguntas y justificar la relación entre variables medibles, este método fue diseñado para mostrar una manera sistemática, precisa y lograr la identificación de las características del fenómeno observado. Los resultados demostraron que la combinación de cal aérea con piedra caliza 
triturada granulada y molida con una relación aglutinante/agregado (a/a) de 0,5 en volumen pueden producir morteros con una resistencia mecánica de 150 días de 18 $\mathrm{kg} / \mathrm{cm} 2$. Esto es dos veces más la resistencia de un mortero equivalente preparado con cal hidráulica y agregado natural. Los resultados mostraron que es posible incorporar APC (agregado reciclado) en morteros de cal para la restauración arquitectónica

Palabras clave: morteros históricos; reciclado; adición puzolánica; toba volcánica; compresión; resistencia. 


\title{
Use of Ignimbrite stone as a substitute for fine aggregate in lime mortars for restoration
}

\begin{abstract}
This research evaluated the use of ignimbrite stone as recycled aggregate and pozzolanic addition in the preparation of lime mortars for architectural restoration. The effect of the compressive strength of traditional mortars was studied, where a part of the traditional binder is replaced by stone dust, and the common aggregate by stone residues. Two methodologies were used, one covers the characterization of the materials involved in the mortars and the other deals with the experimentation of them. The quantitativedescriptive method was used, which allows answering questions and justifying the relationship between measurable variables, this method was designed to show a systematic, precise way and achieve the identification of the characteristics of the observed phenomenon. The results demonstrated that the combination of aerial lime with granulated and ground crushed limestone with a binder/aggregate ratio (b/a) of 0.5 by volume can produce mortars with a mechanical resistance of 150 days of $18 \mathrm{~kg} / \mathrm{cm} 2$. This is twice the strength of an equivalent mortar prepared with hydraulic lime and natural aggregate. The results show that it is possible to incorporate APC (quarry stone sand) in lime mortars for architectural restoration.
\end{abstract}

Keywords: historic mortars; recycled; pozzolanic addition; volcanic tuff; compression; resistance.

Artículo recibido: 02 noviembre. 2021 Aceptado para publicación: 28 noviembre 2021 Correspondencia: donaji.perez.d@gmail.com Conflictos de Interés: Ninguna que declarar 


\section{INTRODUCCIÓN}

En el quehacer arquitectónico y cultural, los morteros de cal son un recurso ampliamente aceptados por los profesionales de la construcción al tener distintas virtudes comparadas con contra el cemento Portland y derivados; los morteros de cal permiten la transpiración de las fabricas donde son colocados, su composición química permite la retracción en la formación de sustancias inestables que pueden ser dañinas para las zonas de trabajo (Arizzi \& Cultrone, 2012). Por lo que es importante tener en cuenta diversas consideraciones en función de su destino; la normativa indica que, en material patrimonial, resguardado por el INAH, se emplee como material cementante pues las dimensiones de los edificios son generoso y robustos. El uso del cemento Portland al ser tan rígido y poco conlleva algunos problemas vinculados a la aparición de sales, incompatibilidades, formación de compuestos expansivos, y módulos no compatibles con las estructuras tradicionales de mampostería y tierra cruda. (Rosell \& Bosch, 2018).

La conservación de edificios catalogados como patrimonio histórico es un tema complejo y trascendente, uno de sus ejes se enfoca en la utilización de morteros para la restauración, (Gisbert Aguliar, Mateos Royo, \& Somovilla de Miguel, 2015); es cierto, que la materia prima actual ya no presenta las mismas características al material original de las edificaciones históricas, lo que origina que en estos procesos se utilicen materiales industriales para mejorar y proteger los sistemas antiguos. Dentro de los estudios sobre alteración de la piedra que se observan de manera recurrente en los edificios históricos, el glosario de ICOMOS (Sites, 2011) hace mención de casos comunes sobre la presencia de agrietamientos y/o desprendimientos de los recubrimientos que son colocados como un método de "protección de sacrificio" en las estructuras pétreas.

Debido al tipo de muros que perduran en los edificios históricos, que en su mayoría están construidas con piedra "cantera" (toba volcánica) absorben el agua proveniente del suelo por la acción capilar evaporándola a través del muro hacia el exterior, arrastrando con ello partículas de la estructura sin afectarla; sin embargo, al existir una barrera (morteros o revestimientos impermeables) que impide la evaporación origina daños en las superficies; entre las más comunes se encuentran los desprendimientos de los aplanados tras condensar el agua en su zona de contacto con la fachada causando daños en las superficies de las superficies que regularmente son elaborados con morteros de cementoarena, ocasionando incompatibilidad de materiales. (Figura 1) 
Figura 1. Diagrama de la humedad proveniente del suelo que se evapora por el muro hacia el exterior
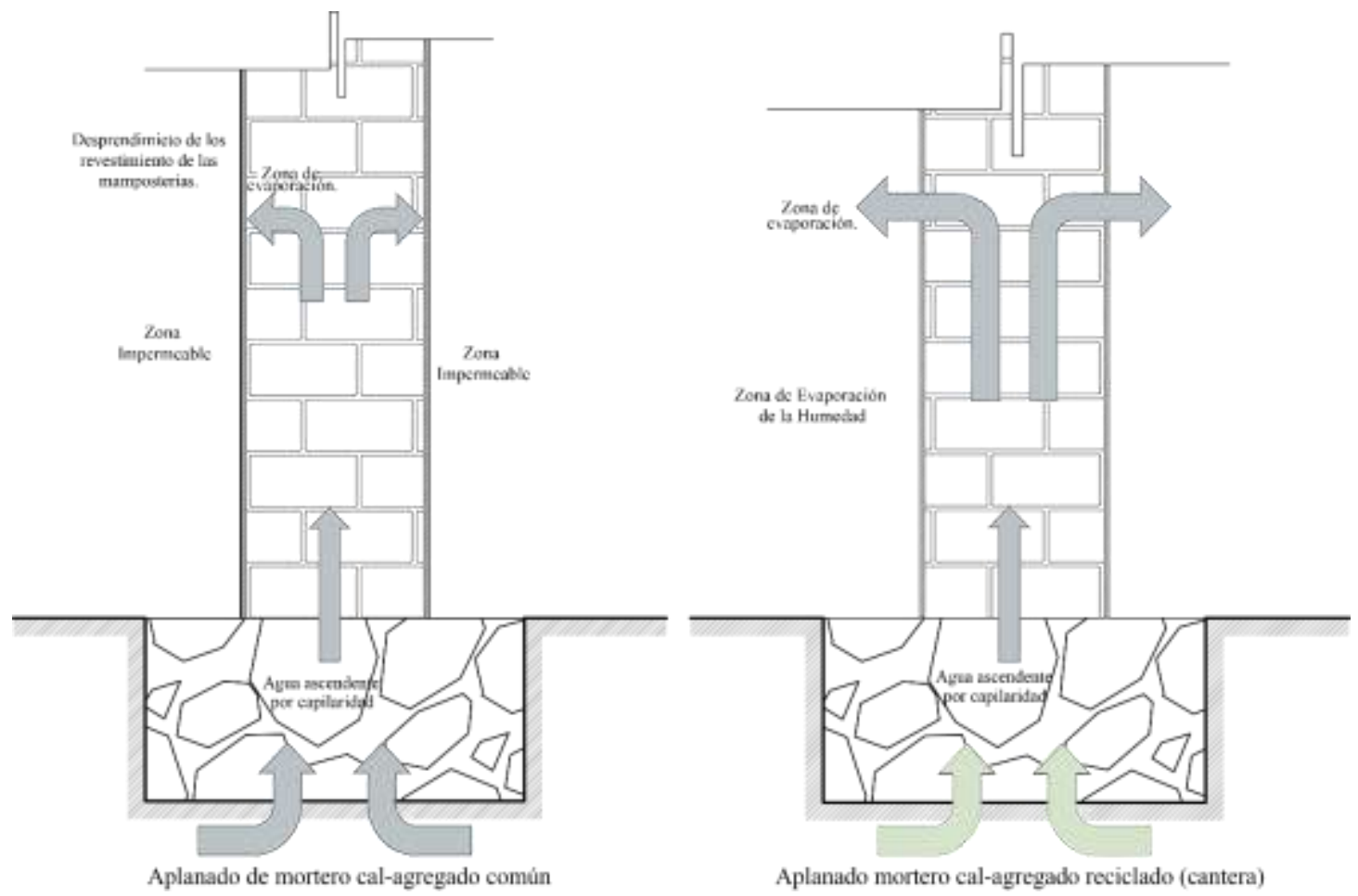

Este experimento se realizó para verificar la factibilidad del uso de piedra triturada como sustituto de la arena en los morteros base cal y cuantificar la resistencia contra los morteros de cal tradicionales para ser usados en procesos de intervención arquitectónica; para ello, se debe considerar el cumplimiento de las demandas funcionales de los elementos de construcción donde se incorporarán morteros nuevos. Se han realizado investigaciones encaminadas al análisis, formulación de nuevos y mejores morteros que puedan contrarrestar los daños, enriqueciendo de esta forma sus propiedades para permitir la conservación de la forma física de la estructura original del inmueble histórico (Matias, Faria, \& Torres, 2014). La cal en pasta posee propiedades cuando entra en contacto con el aire (proceso llamado carbonatación); al carbonatarse, se reconstituye en carbonato de calcio $\left(\mathrm{CaCo}_{3}\right)$, convirtiéndose en la piedra original, no en una masa endurecida compuesta por partículas pegadas entre sí, lo que proporciona plasticidad, permeabilidad y mayor protección a la fábrica original del inmueble. Los morteros de cal de los edificios históricos que existen hasta la actualidad tienen composiciones diversificadas tanto en proporciones, naturaleza, tipo de adiciones y calidad de los componentes principales. (Veiga, 2017) 
El centro histórico de la Ciudad de Oaxaca fue declarado como Patrimonio Mundial por la UNESCO en 1987, y bajo estas disposiciones actualmente los inmuebles se encuentran catalogados: las fachadas pintadas con colores establecidos por la autoridad municipal, colores combinados con la piedra braza de las canteras en sus diferentes tonalidades realzan y conforman una imagen urbana. En él convergen decenas de inmuebles históricos; sin embargo, se ven vulnerables con el paso de los años presentando cambios tanto físicos como químicos en su estructura, lo que da paso a la ejecución de procesos de intervención con la intensión de conservarlos (Terán Bonilla, 2004).

Poco a poco, la investigación de materiales y sistemas constructivos ha conducido a la selección y utilización de diversos residuos para su experimentación, hay estudios donde se ha empleado fibras naturales o de procedencia animal, (Alvarez, 2005) (Waste, J. Ball, \& Paine, 2019) encaminados a mejorar las propiedades de la cal; producto que ha caído en desuso tras la generalización del cemento Portland. Se ha incrementado la concientización sobre el cuidado del ambiente, diversos investigadores han dirigido sus trabajos a la investigación y la reutilización de residuos de algunas industrias aprovechamiento de residuos como materia prima. (Francisco \& Sanchez, 2009; Jiménez, Ayuso, Fernandez, \& de Brito, 2013; Betancourt, Lizarraga, Narayanasamy, Olguin, \& Sáenz, 2015; Waste, J. Ball, \& Paine, 2019).

Ante lo expuesto anteriormente, se hace evidente que la realización de estudios sobre materiales y procesos de restauración de edificaciones históricas apoyados con base en arquitectura tecnológica son imprescindibles; contar con los valores y resultados de este tipo de investigaciones brindará a los profesionales de la restauración arquitectónica herramientas para realizar intervenciones de edificaciones de alto valor patrimonial tomando en cuenta los efectos antes, durante y después de concluir la intervención. A través de la investigación sobre el uso de residuos de piedra cantera como adición puzolánica y como sustitución de arena para la elaboración de morteros de cal será posible generar información útil para que profesionales involucrados en la conservación de los edificios históricos en la Ciudad de Oaxaca, cuenten con alternativas científicas en sus propuestas de intervención lo que podrá minimizar efectos negativos para el inmueble a intervenir. 
Pérez Díaz y otros...

\section{ESTRATEGIAS METODOLÓGICAS O MATERIALES Y MÉTODOS}

\section{A) Aglutinante}

La cal es el material cementante principal utilizado en los procesos de restauración arquitectónica, además de diversos usos en el sector de la construcción. Para este desarrollo experimental se utilizaron dos presentaciones de cal: la cal en pasta $((\mathrm{Ca}(\mathrm{OH}))$; fue proporcionada por el distribuidor, presenta una pureza de $96 \%$ de pureza y 18 meses de añejamiento para este estudio se denominará $(C O X)$. Otro aglutinante utilizado fue cal viva pulverizada $(\mathrm{CaO})$ la cual también fue proporcionada por el fabricante, dicho aglutinante está constituido por partículas ultra finas y $95 \%$ de pureza, y fue apagada en condiciones de laboratorio siguiendo las indicaciones del fabricante; a través del método de apagado tradicional, dejándola a seis meses de añejamiento, para este desarrollo experimental se llamará (CPZ). Finalmente, la cal que se expende comercialmente presenta una pureza del $72-82 \%$ que es utilizada ampliamente en la industria de la construcción fue adquirida en un centro de distribución comercial, se identifica como $(C A H)$. En la Figura 2 se ilustra la procedencia de los materiales utilizados en esta investigación.

\section{B) Agregado}

Los agregados pétreos, que en ocasiones provienen del río, playa, cantera, mina, procesos de trituración, etc. y otras veces son obtenidos a través de la explotación de canteras, cuyo tamaño debe estar comprendido entre 0.075 y $5 \mathrm{~mm}$; son materiales de suma importancia para la elaboración de morteros, por lo que es importante revisar la calidad de los mismos. Su función principal es rellenar y distribuir la retracción durante el secado de los morteros además de proporcionar color, textura, estabilidad volumétrica, resistencia. Deben ser duros, perdurables y limpios (eliminar las impurezas que puedan ser perjudiciales para la estabilidad del mortero, como las orgánicas o los suelos).

El agregado común utilizado en esta investigación proviene de la zona ubicada en San Andrés Ixtlahuaca, Oaxaca con coordenadas $17^{\circ} 04^{\prime} 00^{\prime \prime} \mathrm{N} 96^{\circ} 49^{\prime} 00^{\prime \prime} \mathrm{O}$ (Figura 2). Presentaba una textura granular redondeada en color café lo que garantizaba su idoneidad como componente de los morteros con el tamaño de grano estándar Arena Común $(A C)$. Para este estudio se define a la arena de río como agregado común. El agregado reciclado: árido optimizado Arena de Piedra Cantera $(A P C)$, que para este desarrollo experimental 
se define como agregado reciclado, proviene de un banco de extracción situado en el Municipio de La Soledad, Etla con coordenadas $17^{\circ} 10^{\prime} 01^{\prime \prime} \mathrm{N} 96^{\circ} 49^{\prime} 01^{\prime \prime} \mathrm{O}$ (Figura 2).

Tabla. 1 Composición química de los materiales. * (Amaral, Girondi, Nicolite, \& Teixeira, 2020) Fuente. Por los autores

\begin{tabular}{|c|c|c|c|c|c|c|c|c|c|c|c|c|}
\hline Muestra & $\begin{array}{c}\text { Densidad } \\
\left(\mathrm{g} / \mathrm{cm}^{3}\right)^{*}\end{array}$ & $\mathrm{Al}_{2} \mathbf{O}_{3}$ & $\mathrm{CaO}$ & $\mathrm{Fe}_{2} \mathrm{O}_{3}$ & $\mathbf{K}_{2} \mathbf{O}$ & MgO & $\mathrm{Na}_{2} \mathrm{O}$ & PXC & $\mathrm{SiO}_{2}$ & $\mathbf{P}_{2} \mathbf{O}_{5}$ & MnO & $\mathrm{TiO}_{2}$ \\
\hline Agregado Reciclado & 2.22 & 11.85 & 1.91 & 1.56 & 5.41 & 0.50 & $<0.03$ & 9.05 & 69.59 & $<0.02$ & 0.01 & 0.14 \\
\hline Agregado común & 2.83 & 13.17 & 1.67 & 1.72 & 2.83 & 0.39 & 3.45 & 1.22 & 74.35 & - & - & - \\
\hline
\end{tabular}

Figura 2. Ubicación de los materiales utilizados en el desarrollo experimental

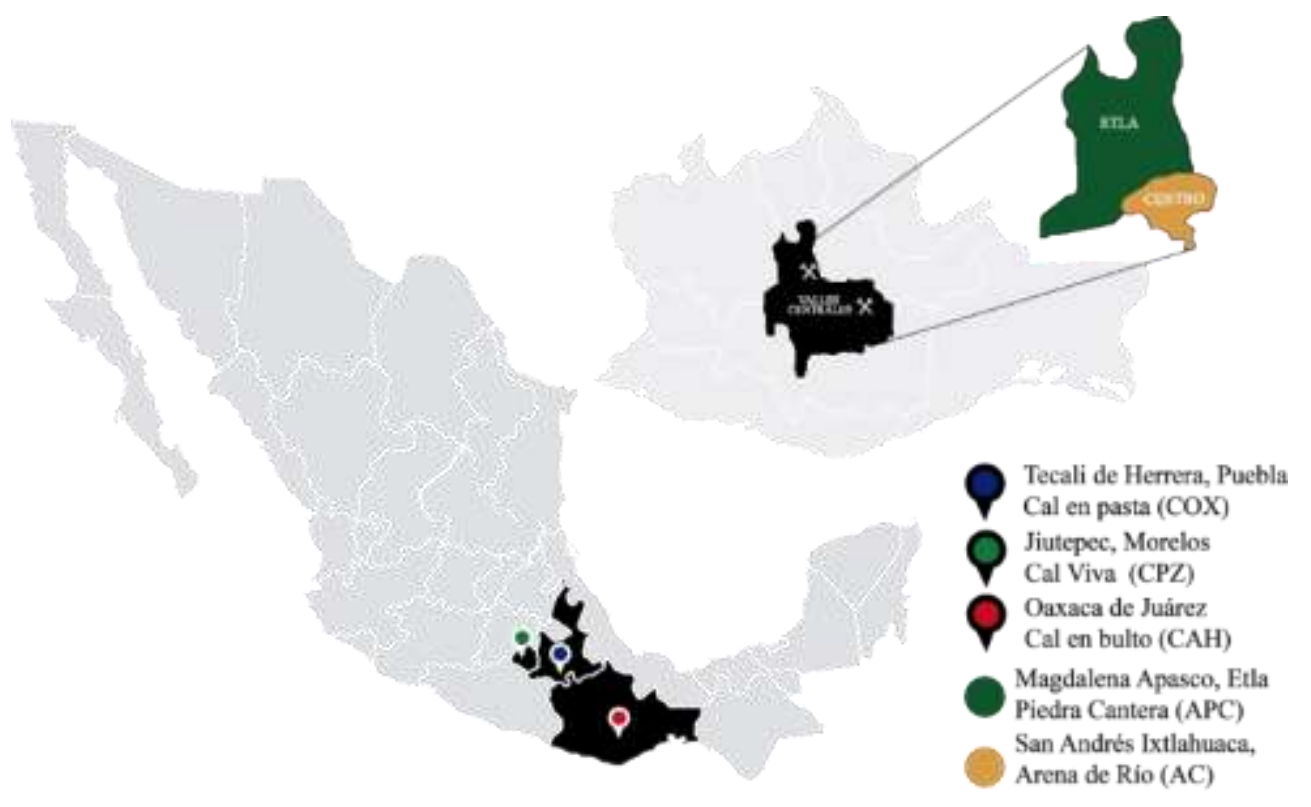

Fuente: Los autores

El agregado reciclado fue obtenido como desecho producto de la explotación de toba volcánica en los bancos de las zonas aledañas a la ciudad, su composición química está conformada en su mayoría por óxido de silicio $\left(\mathrm{SiO}_{2}\right)$ y alúmina $\left(\mathrm{Al}_{2} \mathrm{O}_{3}\right)$ (Tabla 1$)$; este residuo es aprovechado como materia prima para otra industria; sin embargo, la recolección de este material no sigue un proceso estandarizado por lo que los tamaños de las partículas obtenidas en una muestra se encuentran dispersas, algo benéfico pues las granulometrías heterogéneas presentan menores porcentajes de vacíos a ser ocupados por el cementante. Para ambos casos se seleccionaron los finos correspondientes a los que pasan las mallas \#4 (Materials, 2001) hasta la malla \#200, por lo que se realizó un estudio granulométrico para conocer la distribución de las partículas en una muestra 
representativa a fin de obtener un agregado reciclado bien graduado para lograr un mejor empaquetamiento de las partículas dentro de la mezcla del mortero de cal

Tabla. 1 Composición química de los materiales. Fuente. Por los autores

\begin{tabular}{cccccccccccc} 
Muestra & $\mathrm{Al}_{2} \mathbf{O}_{3}$ & $\mathbf{C a O}$ & $\mathrm{Fe}_{2} \mathbf{O}_{3}$ & $\mathbf{K}_{2} \mathbf{O}$ & $\mathbf{M g O}$ & $\mathbf{N a}_{2} \mathbf{O}$ & $\mathbf{P X C}$ & $\mathbf{S i O}_{2}$ & $\mathbf{P}_{2} \mathbf{O}_{5}$ & $\mathbf{M n O}$ & $\mathbf{T i O}_{2}$ \\
\hline Agregado Reciclado & 11.85 & 1.91 & 1.56 & 5.41 & 0.50 & $<0.03$ & 9.05 & 69.59 & $<0.02$ & 0.01 & 0.14 \\
\hline Agregado común & 13.17 & 1.67 & 1.72 & 2.83 & 0.39 & 3.45 & 1.22 & 74.35 & - & - & - \\
\hline
\end{tabular}

\section{Diseño de Mezcla}

Para el trabajo experimental; se seleccionó un sistema de unión, utilizado a menudo en trabajos de restauración arquitectónica, ocupando cal hidratada $(\mathrm{C})$; y agregado natural (AC/APC) en relación aglutinante/agregado, 1/2 y 2/1 (por volumen) y una combinación de cal-adición puzolánica en una relación 0,$10 ; 0.12 ; 0.20$ (en peso) para cada uno de los sistemas aglutinantes.

Los morteros con arena reciclada mostraron una demanda de agua significativamente mayor debido al porcentaje de absorción que posee la piedra cantera, por lo que se tuvo en cuenta a la hora de determinar la relación agua efectiva (disponible) en el aglutinante. Se realizó un estudio previo, con el fin de conocer las proporciones que se usarían respecto a la cal-arena-agua; sin embargo, no existe un método de diseño de morteros o normas que indiquen las proporciones óptimas para la elaboración de los morteros de cal. Las proporciones se establecieron por volumen, como se realizan in situ; es decir, para la relación 1:1, corresponde a 1 parte de la cal en pasta por una parte de arena. Los aspectos tomados para saber qué proporción es la más adecuada fue la fluidez, se retomaron de la norma NMX-C-061-ONNCCE-2010, las mezclas de mortero se diseñan con dos fines, uno de ello es para tener buenas prestaciones mecánicas y de durabilidad, y el segundo objetivo busca una mezcla que atienda a valores económicamente factibles.

La adición puzolánica (polvo de piedra cantera) que se utilizó es resultado de la trituración por medio del molino y el tamaño de la partícula debe pasar la malla \#200 (0.074 mm). El propósito de utilizar el polvo de la piedra cantera como sustitución de la cal es para incrementar las propiedades de los morteros elaborados con cal y agregado reciclado; es decir, reemplazar un porcentaje del contenido de la cal por polvo de piedra cantera. Se establecieron sustituciones de $0.10,0.12$ y $0.25 \%$ (tabla 2 ) con respecto al peso de la cal, estos porcentajes se definieron con base a estudios previos de las mezclas, se observó que estos proporcionan homogeneidad y trabajabilidad a la mezcla. 
Tabla 2. Proporciones de mezclas de los morteros. Fuente: Por los autores

\begin{tabular}{ccccc}
$\begin{array}{c}\text { Componentes } \\
\text { del mortero }\end{array}$ & $\begin{array}{c}\text { Cal }(\mathbf{C}) \\
(\mathbf{k g})\end{array}$ & $\begin{array}{c}\text { Arena de río } \\
(\mathbf{A R})(\mathbf{k g})\end{array}$ & $\begin{array}{c}\text { Arena } \\
\text { optimizada } \\
(\mathbf{A P})(\mathbf{k g})\end{array}$ & $\begin{array}{c}\text { Adición } \\
\text { Puzolánica } \\
(\mathbf{P P C})(\mathbf{k g})\end{array}$ \\
\hline $\mathbf{M - 0 1}$ & 0.7934 & 1.8972 & 1.1574 & - \\
\hline $\mathbf{M - 0 2}$ & 1.5868 & 1.8972 & 1.1574 & - \\
\hline $\mathbf{M - 0 3}$ & 0.7934 & 1.8972 & 1.1574 & 0.0793 \\
\hline $\mathbf{M - 0 4}$ & 1.5868 & 1.8972 & 1.1574 & 0.0793 \\
\hline $\mathbf{M - 0 5}$ & 0.7934 & 1.8972 & 1.1574 & 0.0922 \\
\hline $\mathbf{M - 0 6}$ & 1.5868 & 1.8972 & 1.1574 & 0.0922 \\
\hline M-07 & 0.7934 & 1.8972 & 1.1574 & 0.1983 \\
\hline M-08 & 1.5868 & 1.8972 & 1.1574 & 0.1983 \\
\hline Relación agua/aglutinante $(\boldsymbol{\%}) \mathbf{0 . 8 1}$ & & & \\
\hline
\end{tabular}

\section{DESARROLLO}

\section{Caracterización Granulométrica}

\section{A) Arena Común o de Río (AC)}

La dimensión de las partículas del agregado tiene una influencia decisiva en la estructura porosa y el subsecuente comportamiento de un mortero, la caracterización de esta propiedad recurre a la determinación de las fracciones de arena cuyas partículas tienen una dimensión definida entre dos límites pre-definidos; la granulometría se designa a la distribución de estas fracciones y generalmente está representada por un gráfico denominado curva granulométrica (Chan Yam, Solís Carcaño, \& Moreno, 2003).

\section{B) Piedra Cantera $(A P C)$}

Como se ha mencionado anteriormente; se seleccionó la piedra llamada ignimbrita con el objetivo de evaluar la efectividad de su utilización como agregado principal en la elaboración de morteros de cal para restauración arquitectónica, por lo que es indispensable cumplir con los requerimientos granulométrico. De igual manera se seleccionó polvo de piedra cantera (PPC) que pasara la malla \#200, con el fin de utilizarlo como adición puzolánica en la elaboración de morteros de cal; la prueba de granulometría se realizó con los mismos parámetros del agregado común bajo condiciones de laboratorio, siguiendo las recomendaciones de la norma ASTM C136-05 (International, 2018)

De esta forma se pudo conocer las diferencias entre los dos agregados, al revisar los resultados de las curvas granulométricas se observó que el agregado reciclado mostró una discontinuidad entre la malla \#16 y la malla \#4 lo que sugería una mayor presencia de partículas correspondientes a la malla \#8 $(2.38 \mathrm{~mm})$ lo anterior podría representar una 
irregularidad, al momento de elaborar los morteros por lo que se decidió realizar un proceso de optimización para asegurar la igualdad de condiciones como agregado fino.

Figura 3. Realización de la prueba de granulometría en áridos. Fuente: Por los autores.

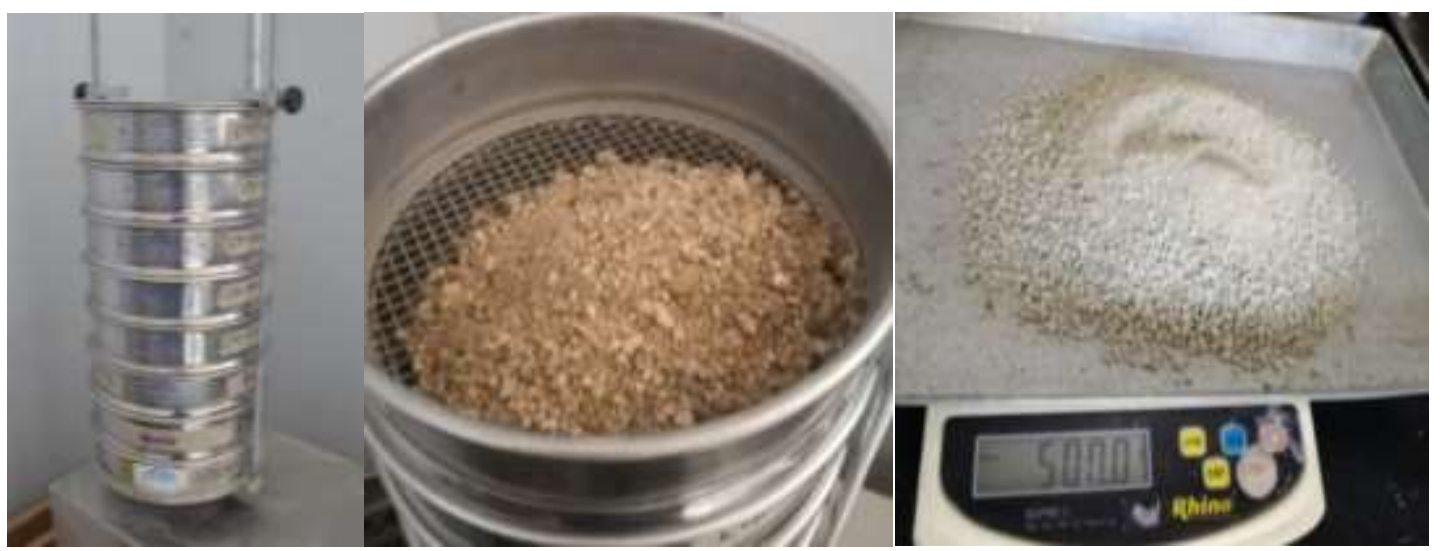

\section{Optimización del Árido Reciclado}

El uso de áridos finos reciclados como materia prima en la elaboración de morteros aparece como una buena alternativa para minimizar la disposición de residuos, reducir el consumo de recursos naturales y encontrar y suministrar sustitutos adecuados de los áridos naturales. (Lopes Lima \& Batista Leite, 2012). Se sabe que las características de los agregados en cuanto a su forma, textura y gradación influyen en la trabajabilidad, en el acabado, en la exudación y en la segregación de los morteros afectando la resistencia, la rigidez, la retracción, la densidad, la permeabilidad y durabilidad de los mismos (Quiroga \& Fowler, 2003).

El proceso de optimización del agregado reciclado (APC) proveniente de la explotación y labra de piedra cantera; se realizó bajo condiciones de laboratorio replicando el volumen de las partículas del agregado común ocupado en una muestra referencial. Dicho volumen fue replicado con el agregado reciclado, reproduciendo cada volumen de los diferentes granos sin considerar su peso hasta completar la muestra total. Al finalizar el proceso de repitió la prueba de granulometría utilizando el agregado común referenciado y el agregado reciclado optimizado; así, finalmente se pudo apreciar una mayor similitud en el comportamiento de la curva granulométrica (tabla 3 y Figura 4). 
Figura 4. Partículas de agregado común y Partículas de agregado reciclado.

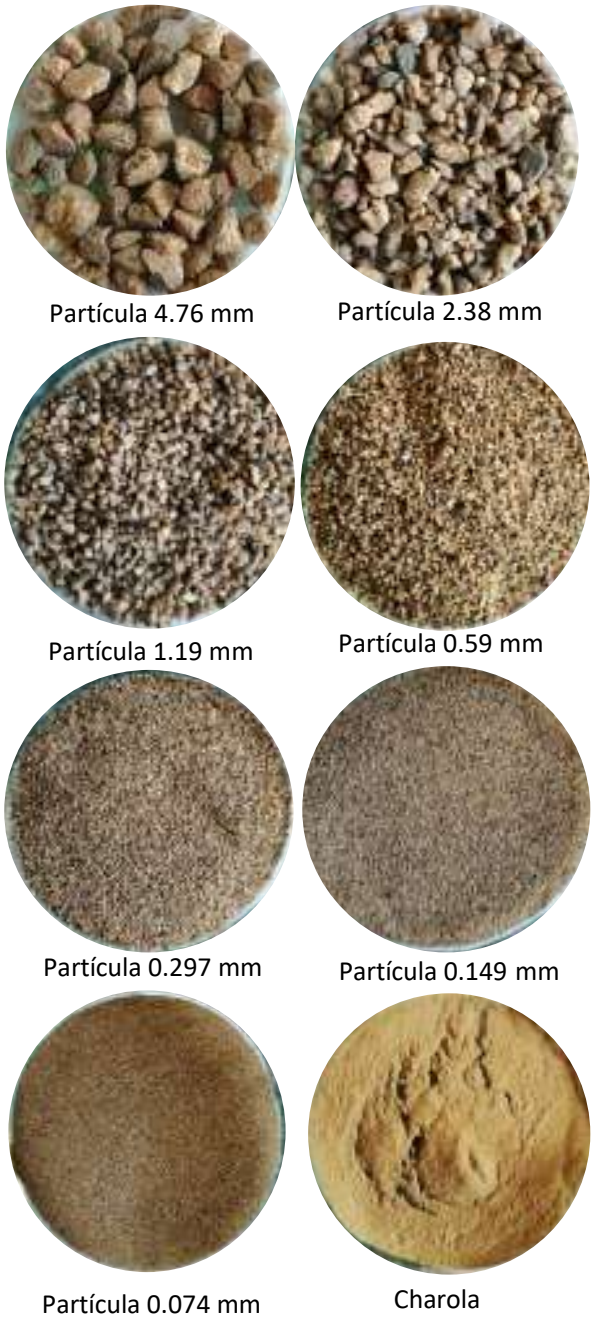

A)

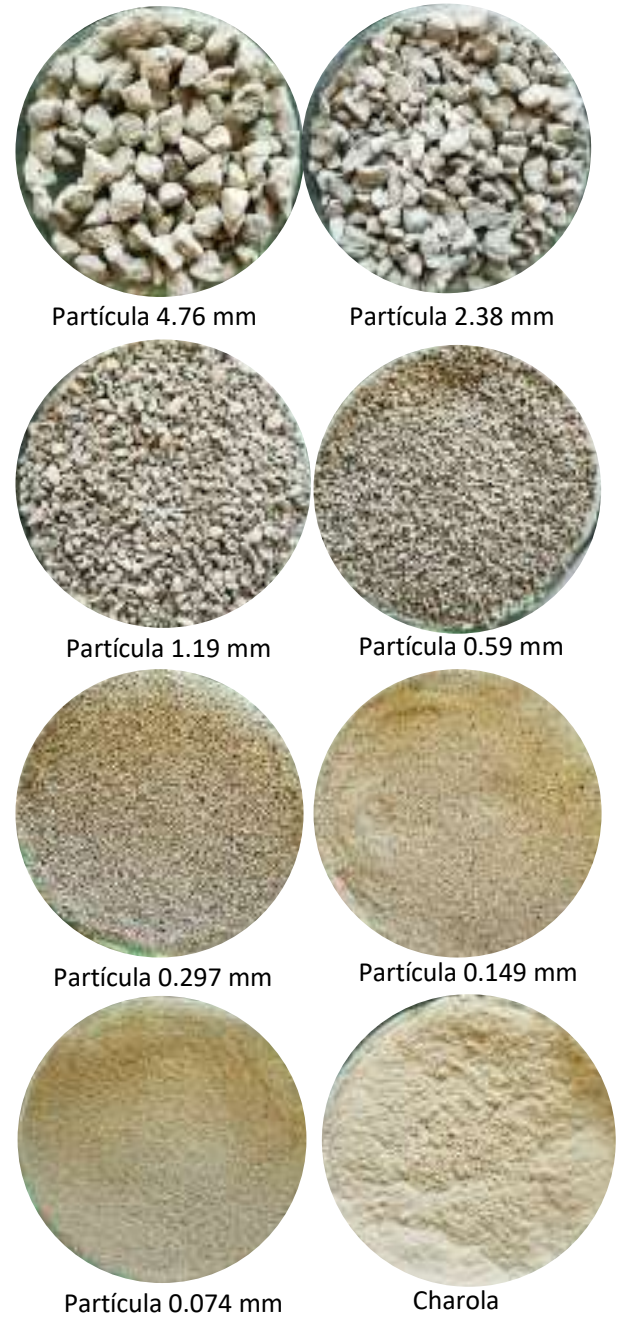

B)

Fuente: Por los autores

Se observó que la muestra optimizada del agregado reciclado (piedra cantera) mostraba un peso inferior en comparación con el agregado común; es decir, a pesar del comportamiento granulométrico similar difieren del peso contenido en la muestra (figura 4). Esto puede estar relacionado con las densidades de los materiales como se presenta en la tabla 3. (Amaral, Girondi, Nicolite, \& Teixeira, 2020) quienes sugieren que la mayor densidad de la arena común puede incrementar el peso final del producto sin contribuir a un aumento de su resistencia específica. 
Tabla 3. Resultados de la optimización granulométrica de la arena de río (AC) y arena de piedra cantera (APC)

\begin{tabular}{|c|c|c|c|c|c|c|c|c|c|}
\hline \# Malla & $\begin{array}{l}\text { Apertura } \\
\text { en }(\mathbf{m m})\end{array}$ & $\begin{array}{c}\text { Peso } \\
\text { retenido } \\
(\mathrm{AC}) *\end{array}$ & $\begin{array}{c}\text { Peso } \\
\text { retenido } \\
(\mathrm{APC}) * *\end{array}$ & $\begin{array}{c}\% \\
\text { retenido } \\
\text { (AC) }\end{array}$ & $\begin{array}{c}\% \\
\text { retenido } \\
\text { (APC) }\end{array}$ & $\begin{array}{c}\% \\
\text { acumulativo } \\
\text { (AC) }\end{array}$ & $\begin{array}{c}\% \\
\text { acumulativo } \\
\text { (APC) }\end{array}$ & $\begin{array}{c}\text { \% Que } \\
\text { pasa } \\
(\mathrm{AC})\end{array}$ & $\begin{array}{c}\text { \% Que } \\
\text { pasa } \\
\text { (APC) }\end{array}$ \\
\hline 4 & 4.76 & 149.50 & 94.80 & 7.91 & 8.17 & 7.91 & 8.17 & 92.09 & 91.83 \\
\hline 8 & 2.38 & 443.20 & 272.20 & 23.45 & 23.47 & 31.35 & 31.64 & 68.65 & 68.36 \\
\hline 16 & 1.19 & 449.00 & 256.00 & 23.75 & 22.07 & 55.11 & 53.72 & 44.89 & 46.28 \\
\hline 30 & 0.59 & 419.70 & 258.70 & 22.20 & 22.31 & 77.31 & 76.02 & 22.69 & 23.98 \\
\hline 50 & 0.297 & 249.80 & 158.80 & 13.21 & 13.69 & 90.53 & 89.71 & 9.47 & 10.29 \\
\hline 100 & 0.149 & 122.50 & 83.00 & 6.48 & 7.16 & 97.01 & 96.87 & 2.99 & 3.13 \\
\hline 200 & 0.074 & 36.80 & 23.50 & 1.95 & 2.03 & 98.95 & 98.90 & 1.05 & 1.10 \\
\hline Charola & & 19.80 & 12.80 & 1.05 & 1.10 & 100.00 & 100.00 & 0.00 & 0.00 \\
\hline Suma & & 1890.30 & 1159.80 & 100.00 & 100.00 & 100.00 & 100.00 & & \\
\hline
\end{tabular}

Fuente: Por los autores

Figura 4. Comportamiento granulométrico: (A) agregado común. (AR) (B) Agregado reciclado posterior a la optimización. (APC)

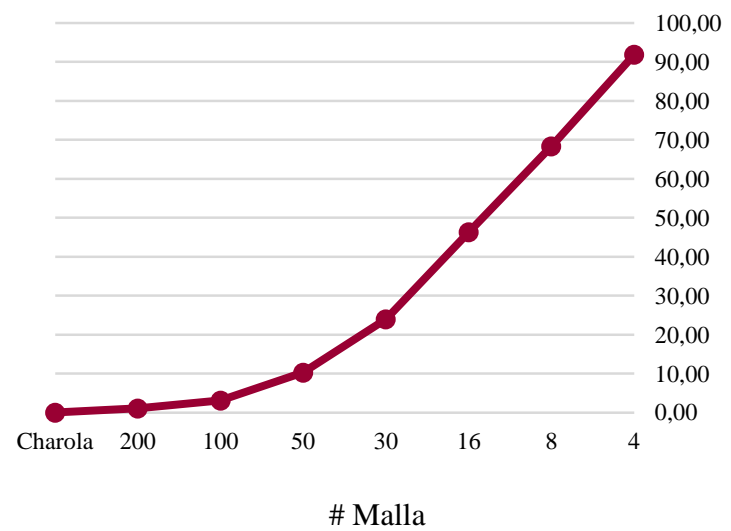

A)

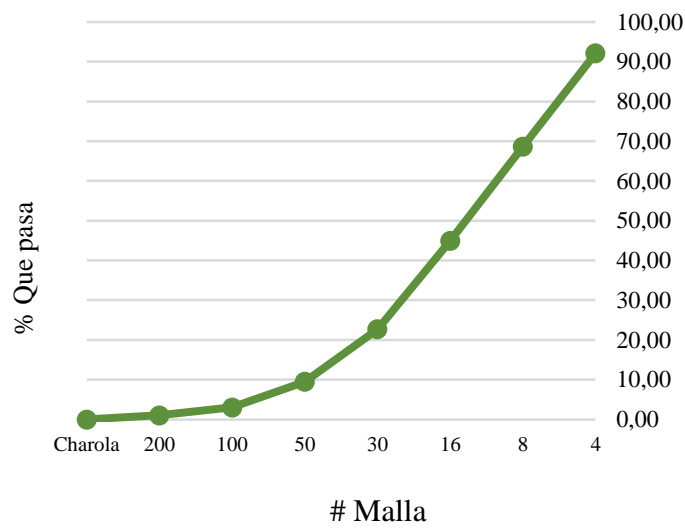

B)

Fuente: Por los autores

\section{Elaboración de Especímenes}

Los morteros frescos fueron colados en moldes cúbicos de 50x50x50 mm para los ensayos de resistencia a la compresión a 90, 120, 150 días. Se confeccionaron 480 especímenes en total con las mezclas previamente establecidas. (tabla 4), la relación agregadoaglutinante (por volumen) fue 1:1 y 2:1. En la tabla 4 se muestran los detalles de las mezclas evaluadas. Dado que no existen normas específicas para la elaboración de morteros de cal se siguieron las indicaciones de la norma para morteros de cemento según NMX-C-159-ONNCCE-2016 (2016). Los especímenes fueron desmoldados al cabo de 72 horas, y curados a temperatura ambiente hasta realizar las pruebas. Inicialmente se pesó el agregado reciclado $(P P C)$ (1 parte) con la graduación establecida en la 
optimización de la granulometría (tabla 3). Al utilizar tres tipos de cal, se realizaron diferentes procesos en la preparación y manufacturación de dichos especímenes.

Para la elaboración de los especímenes con adición puzolánica, se realizó el mismo proceso de selección de materiales; se añadió el polvo de piedra cantera (tamaño 0.074 mm) según los porcentajes establecidos previamente en el diseño experimental (tabla 2). La cal hidráulica $(\mathrm{CAH})$ que se utilizó, fue preparada previamente en forma de pasta siguiendo las recomendaciones del fabricante, con el agregado reciclado $(P P C)$ se confeccionaron 15 especímenes cúbicos para cada tipo de cal en cada mezcla diferente (figura 5). Se estableció un mortero testigo utilizando cal hidráulica-agregado común, manteniendo las características de un mortero utilizado en procesos de intervención arquitectónica.

El procedimiento para el colado de los cubos se realizó de la siguiente manera: se prepararon los moldes cúbicos como se muestra en la figura 6, limpiándolos y lubricándolos con aceite para desmoldarlos posteriormente. Se llenó el molde en dos capas, golpeando 32 veces con un pisón de acrílico cada capa, cada molde se golpeó sobre la superficie de trabajo cinco veces para eliminar el exceso de aire en el mortero. Finalmente se enrasó el molde con una espátula para eliminar excedentes de la mezcla sobre la superficie.

Dado que la cal posee un proceso de fraguado lento en comparación con otros aglutinantes el cemento, no fue posible desmoldar los especímenes a las 24 horas posteriores a su colado por lo que se dejaron envejecer de 3 a 4 días para evitar daños en su desmolde. Una vez desmoldados, se registró su masa inicial del cubo para comparar las masas superficiales de cada mezcla y agregado de los diferentes morteros; posteriormente se resguardaron las muestras en un espacio a temperatura ambiente para comenzar el fraguado de las mismas.

Figura 5. Preparación de los morteros a base de cal-agregado reciclado. Fuente: Por el autor

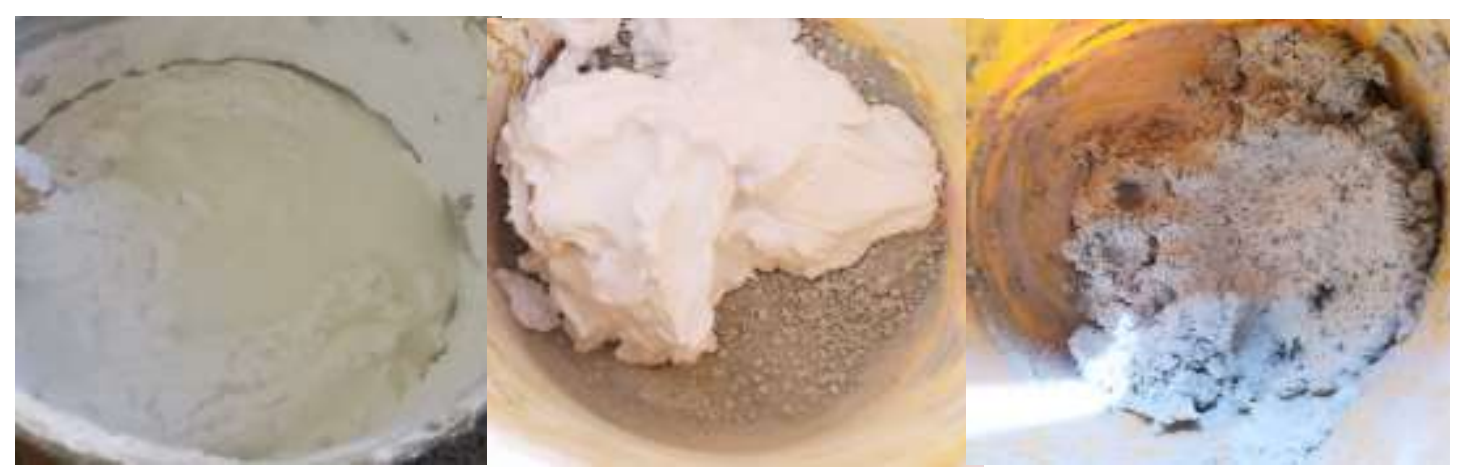


Figura 6. Preparación de los morteros a base de cal-agregado reciclado

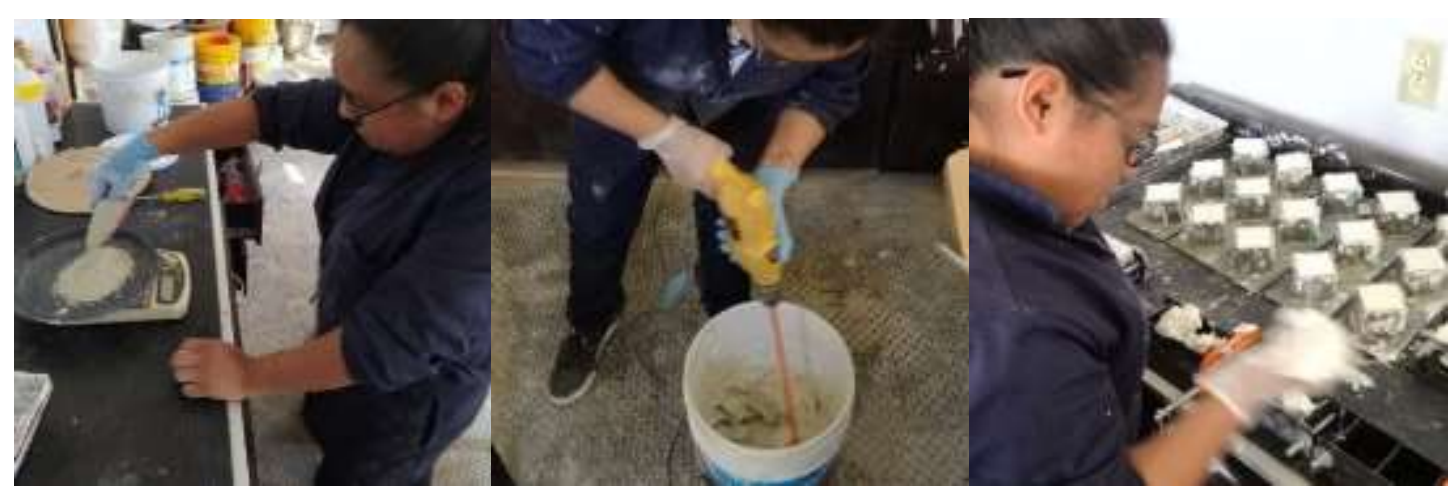

Fuente: Por el autor

\section{Caracterización de los Morteros}

\section{Densidad aparente:}

La densidad es una de las propiedades físicas más importantes en los morteros; es una medida del grado de consolidación de un sólido, así mismo informa del empaquetamiento de granos y la resistencia mecánica. (Pavía \& Treacy, 2006). la cual está directamente relacionada con la densidad de sus materiales componentes, así como el contenido de aire en el mismo. En el mismo sentido a mayor densidad indica que el mortero es más compacto en su interior; por lo tanto, una menor densidad hace referencia a que la estructura de la muestra presenta una cantidad mayor de poros en el interior.

Para el desarrollo de este procedimiento se utilizaron especímenes cúbicos, a lo cuales se les cuantificó la masa inicial una vez desmoldados, y la masa final previo a la prueba destructiva de resistencia a la compresión. A través de una ecuación matemática (1) se pudo conocer la densidad de los morteros elaborados en este programa experimental. En la gráfica 2 se muestran las densidades de los morteros.

$$
\rho=\frac{m}{v}
$$

\section{Donde:}

$\rho=\operatorname{Densidad}\left(\mathrm{g} / \mathrm{cm}^{3}\right)$

$\mathrm{m}=$ Masa seca $(\mathrm{g})$

$\mathrm{v}=$ Volumen $\left(\mathrm{cm}^{3}\right)$ 


\section{Evaluación de la resistencia a la compresión:}

Existen normas nacionales e internacionales que detallan los requerimientos que deben cumplir los diferentes materiales y sistemas constructivos utilizados en la construcción actual. Las normas (UNE) están enfocadas al estudio de los morteros; en su mayoría al cemento, aunque existen normas referentes a la cal.

Respecto a las propiedades mecánicas de los morteros de cal; una de las más importantes es la resistencia a la compresión, la cual, está relacionada con la durabilidad. El mortero endurecido tiene que ser suficientemente resistente y debe desarrollar una adecuada adhesión a las demás unidades de mampostería (Lanas \& Alvarez, 2006). Para esta prueba se prepararon 360 especímenes cúbicos de 50mm x 50mm x 50mm, utilizando las mezclas previamente establecidas en el diseño experimental (Tabla 2). La prueba se realizó en la prensa hidráulica (Controls ASTM Compression Machines), se elaboraron morteros de cal a 90, 120 y 150 días de edad a temperatura ambiente.

La Figs. 2a - h muestran la resistencia a la compresión de los morteros de cal a los 90, 120, 150 días de curado. Los morteros con pasta de cal con agregado reciclado presentan mayores valores de resistencia a la compresión en comparación con los morteros de cal con agregado común. Este hecho puede estar relacionado con el tiempo de añejamiento y la red de poros que presentan los grupos de morteros.

Figura 7. Realización de la prueba de compresión

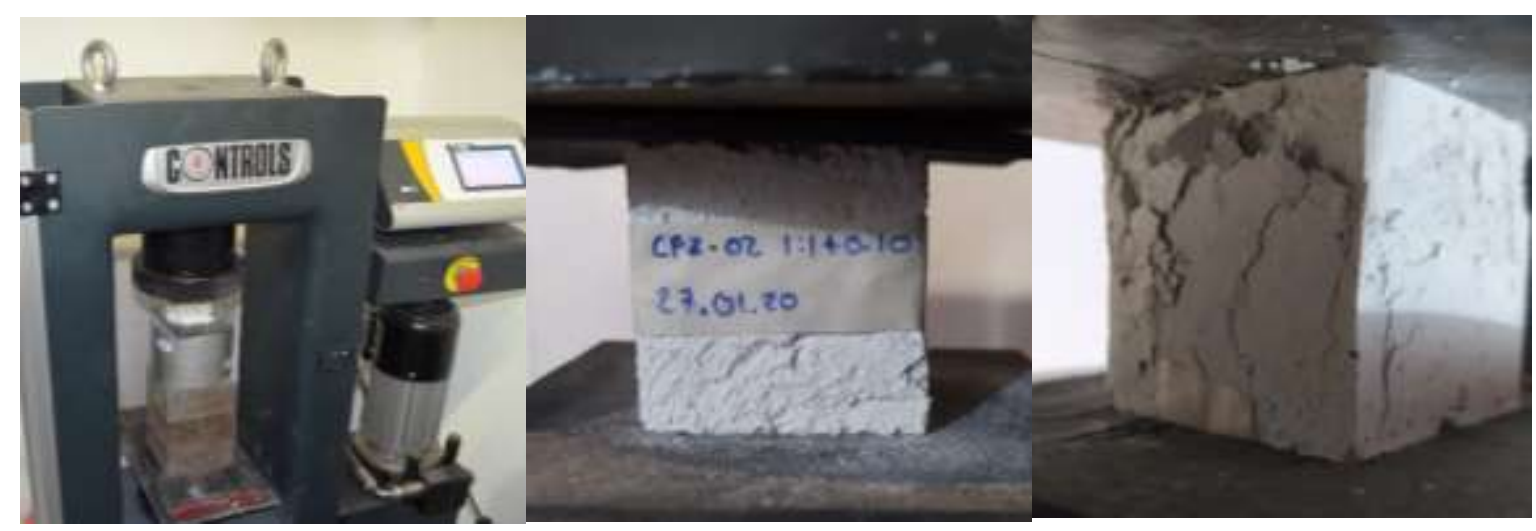

Fuente: Por el autor

\section{RESULTADOS Y DISCUSIÓN}

\section{Densidad aparente:}

En la figura 8 se muestran los resultados de la densidad de los morteros de cal, como se puede apreciar, la mezcla del mortero testigo presenta las densidades más altas, entre 1.40 
$\mathrm{g} / \mathrm{cm} 3$ y $1.80 \mathrm{~g} / \mathrm{cm} 3$, estos resultados tienen relación con la densidad del agregado común (arena) material que cuenta con una densidad mayor que el agregado reciclado proveniente de la piedra cantera. Este resultado también puede indicar que el mortero presenta una estructura más compacta ante la ausencia de poros en su interior.

En contradicción con las densidades de los morteros hechos con la cal $C P Z$, la cual obtuvo densidades más bajas en comparación con las cales $C O X$ y $C A H$, por lo que se puede considerar al mortero $C P Z$ como un mortero ligero dado que su densidad se encuentra en un promedio de $1.16 \mathrm{~kg} / \mathrm{cm}^{3}$; sin embargo, la densidad no se puede integrar en un solo valor, puesto que cada mezcla tiene diferente composición.

Se puede observar que las cales $C O X$ y $C A H$, mostraron un comportamiento similar entre sí, puede verse que el agregado reciclado impacta en la estructura física de los morteros, se sabe que la piedra cantera es una estructura con vacíos en su matriz mineral . (Amaral, Girondi, Nicolite, \& Teixeira, 2020) sugieren que la mayor densidad de la arena puede incrementar el peso final del producto sin contribuir a un aumento de su resistencia específica.

(Klemm, 2016) indica que las piedras de construcción sedimentarias (arenisca, caliza) son susceptibles a los agentes de descomposición. Sin embargo, las estructuras patrimoniales construidas con estas dos piedras exhiben una longevidad notable. Por lo que se infiere que la utilización de un agregado con estas propiedades otorgaría durabilidad a los morteros de los edificios históricos de piedra. Pero se requiere realizar experimentaciones relacionados a la durabilidad de los morteros.

Figura 8. Densidad de los morteros

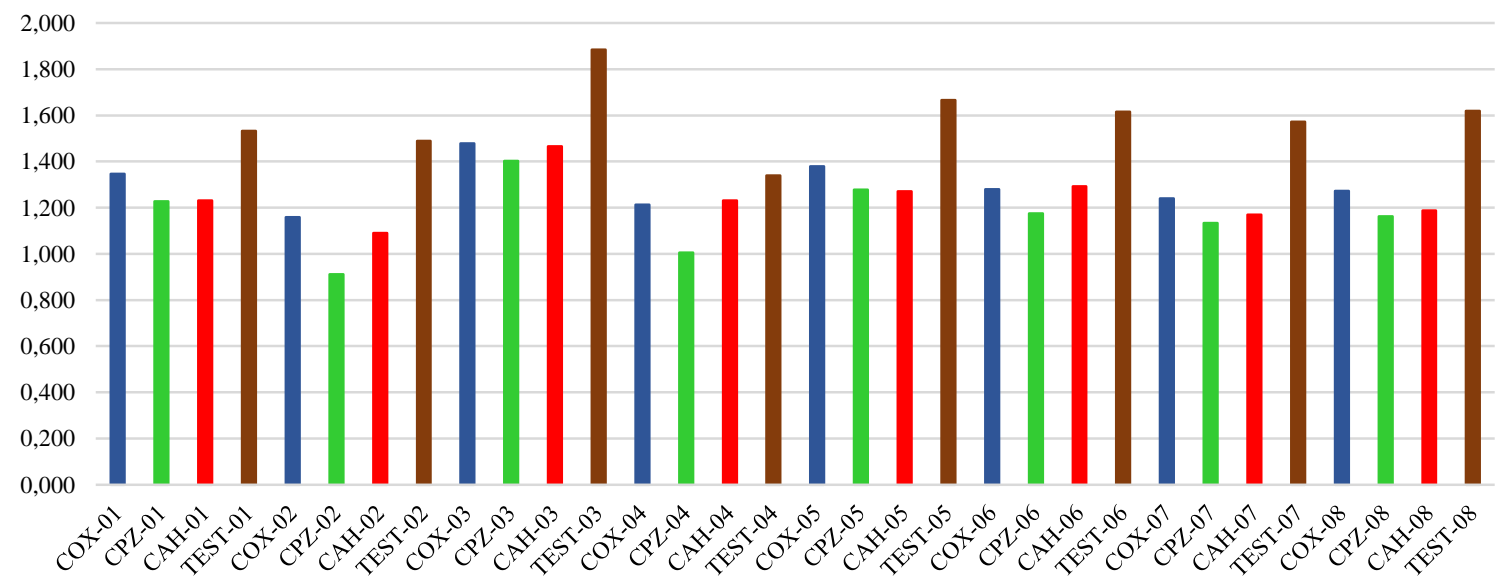

Fuente: Por los autores 


\section{Evaluación de la resistencia a la compresión:}

El desarrollo de la resistencia a la compresión de los morteros se muestra en la figura 9a -9h. Puede verse que la arena reciclada no obstaculiza el desarrollo de la resistencia; por el contrario; en los morteros de cal blandos, la arena reciclada parece mejorar la resistencia en las edades tempranas. Se puede apreciar que las mezclas correspondientes a la cal (COX-01) registraron las mayores resistencias, este comportamiento fue más evidente en los morteros de la mezcla de cal-agregado reciclado (Fig. 9a) al obtener resistencias de $22.57 \mathrm{~kg} / \mathrm{cm}^{2}$ a los 150 días de edad; por lo tanto, fue la mezcla que alcanzó las mayores resistencias. Mientras que los morteros de cal-agregado común registraron resistencias de $12.64 \mathrm{~kg} / \mathrm{cm}^{2}$ a la misma edad. Se puede apreciar que cada mezcla y cada tipo de cal mostró un desarrollo de resistencia diferente entre sí. La cal (COX-01) fue la que obtuvo mayores resistencias en comparación con las otras cales, obteniendo hasta un $46 \%$ superior al resto de las mezclas, incluso el mortero testigo (TEST-01) con agregado común la cual obtuvo resistencias similares al mortero de la cal (CAH-O1) y agregado reciclado.

Las figuras 9c y 9d muestran el desarrollo de resistencia de las mezclas de cal-agregadopuzolánica (polvo de piedra cantera); en el caso de la mezcla 03, se observa que la cal $C O X$ alcanzó resistencias superiores, las cales $C P Z$ y $C A H$ mostraron un desarrollo de resistencia similares entre sí. Por su parte, la mezcla 04 registró resistencias superiores que la mezcla 03 en todos los tipos de cales; esto se pudo haber originado por el contenido de calcio en el mortero; dado que los morteros de la mezcla 03 cuenta con una mayor cantidad de hidróxido de calcio: la pasta de cal y el polvo de piedra como adición puzolánica en su estructura. Estas condiciones de la mezcla pudieron favorecer en el desarrollo de la resistencia de los morteros. 
Figura 9. Resultados de la prueba de compresión. Fuente: Por los autores

9a
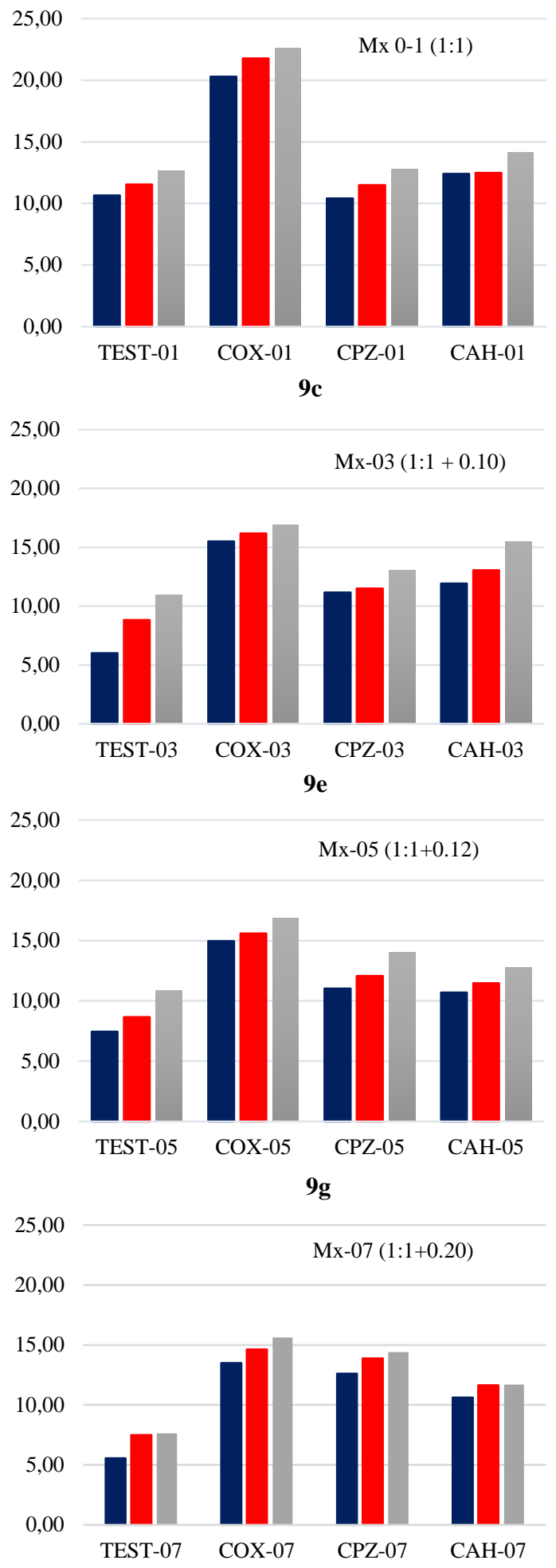

9b
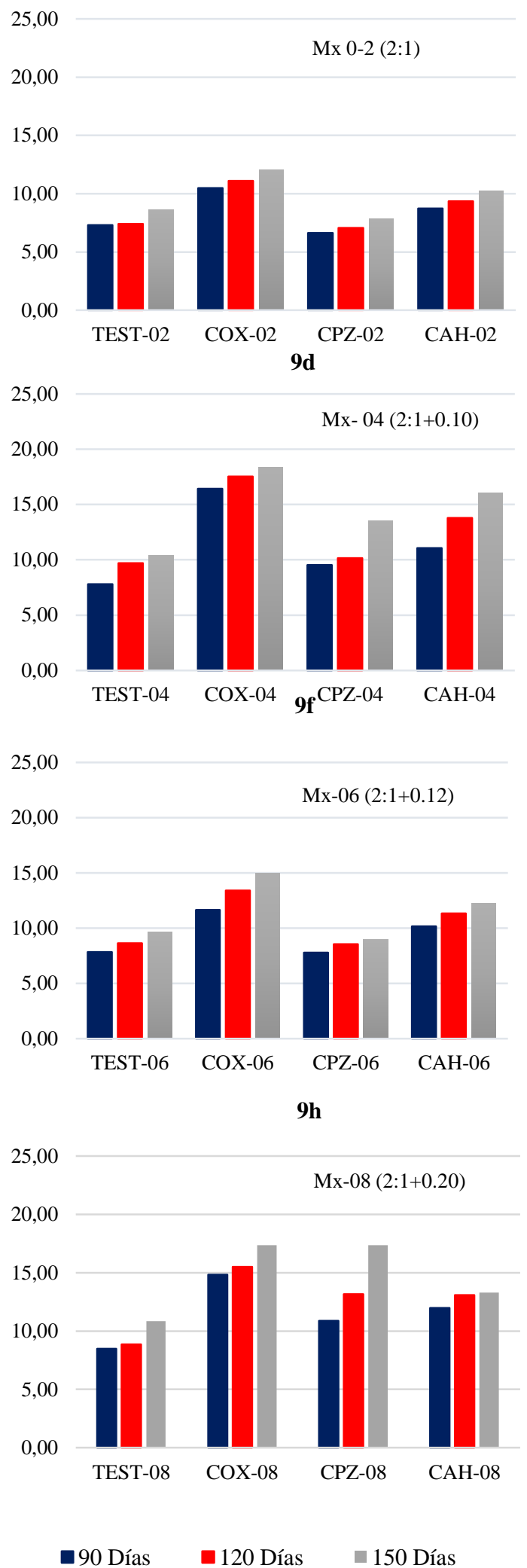
Por su parte, las mezclas 05 y 06 mostraron un desarrollo de su resistencia parecido a la mezcla 04, esto pudo ser causado por la relación del contenido de la adición puzolánica en la mezcla, la cual aumentó $2 \%$ con respecto a la mezcla 05 . Como se observó, la cal tipo COX-05 y COX-06 mostraron mayores resistencias que el resto de las preparaciones alcanzando hasta $15 \mathrm{~kg} / \mathrm{cm} 2$ a los 150 días de envejecimiento; las cales CPZ-05 y CAH05, presentan un comportamiento afín entre las dos; alcanzando $13.49 \mathrm{~kg} / \mathrm{cm} 2$ y 16.04 $\mathrm{kg} / \mathrm{cm} 2$ a los 150 días respectivamente; sin embargo, el mortero preparado con la tal tipo $\mathrm{CAH}$ y agregado reciclado obtuvo mejores prestaciones que el mortero testigo preparado con agregado común el cual alcanzó $10.85 \mathrm{~kg} / \mathrm{cm} 2$ de resistencia a la compresión.

Los morteros correspondientes a las mezclas 07 y 08 presentaban el mayor porcentaje de adición puzolánica (polvo de piedra cantera) aumentando la cantidad de óxido de calcio de la pasta involucrada en la mezcla, por lo que se esperaba mayor desempeño mecánico que el resto de las mezclas; sin embargo, el efecto de dicha adición no se vio reflejada a las edades de prueba, mostrando un comportamiento por debajo de los rendimientos de las demás mezclas.

No obstante, la evaluación de la resistencia a la compresión ilustra una tendencia ascendente, lo que hace reflexionar que la adición puzolánica favorecerá la mezcla en las edades tardías.

Ante esto, se observó que la mezcla 08 obtuvo mayores resistencias que la mezcla 07 , esto pudo ser originado por la cantidad de óxido de cal contenido en el mortero, la mezcla 08 estaba formada por dos partes del aglutinante y una parte del agregado común, con $20 \%$ de adición puzolánica; por lo que se infiere que a mayor cantidad de óxido de calcio en el mortero aumenta su desempeño mecánico. Así mismo se observó que a edades tardías, los morteros alcanzaron las resistencias más altas, como es en el caso de las cales COX-08 y CPZ-08, las cuales obtuvieron la misma resistencia a los 150 días envejecimiento. Por lo tanto, se deduce que los morteros con esta composición alcanzarían mayores resistencias si se dejaran a un envejecimiento más prolongado.

\section{CONCLUSIÓN O CONSIDERACIONES FINALES}

Este estudio evaluó la viabilidad de reemplazar el agregado común por el agregado reciclado procedente de residuos de bancos de explotación de piedra cantera en lo morteros tradicionales a base de cal. El proceso de optimización en laboratorio de la arena 
reciclada $(A P C)$ permitió una distribución de grano uniforme, sin la necesidad de limpiar y adicionar materiales orgánicos.

La absorción de agua debido a la acción capilar del agregado reciclado aumentó considerablemente para las proporciones de reemplazo por encima del 10\%. Los morteros con $A P C$ presentaron mayor demanda de agua y menor trabajabilidad en comparación con los morteros con arenas naturales. La piedra cantera; al ser un material poroso, requirió mayor cantidad de agua para su manejabilidad; a diferencia del agregado común, que presenta una menor capacidad de absorción y por consiguiente menor cantidad de agua para alcanzar la consistencia ideal.

Con base en los resultados de la prueba mecánica se pueden extraer las siguientes conclusiones: la adición de un 12\% de (PPC) tuvo efectos en las propiedades de los morteros: un aumento de la resistencia a la compresión a edades tempranas en comparación con los morteros con el agregado común sin adiciones, de la misma forma el desarrollo de su resistencia se mantuvo en ascenso al cabo de los 150 días.

En los morteros actuaron dos mecanismos; uno pudo ser la alta porosidad (debido al alto contenido de agua y la naturaleza de los agregados) que ayuda a la reacción química debido a la presencia de componentes reactivos al utilizar polvo de piedra cantera como adición puzolánica creando una estructura fuerte. Los resultados fueron alentadores al mostrar un efecto deseable al incorporar APC (arena de piedra cantera) como sustitución del agregado fino en la elaboración de morteros de cal, que son ampliamente utilizados en trabajos de reparación de estructuras históricas sin afectar la resistencia mecánica del mortero.

Las características técnicas de los morteros a base de agregado reciclado en cuanto a resistencia mecánica, no se comparan con los valores que alcanzaron los morteros convencionales de cal utilizados en la actualidad en trabajos de reparación. Esta investigación plantea la posibilidad de reutilizar un agregado en la elaboración de materiales para procesos de restauración, sin embargo, es necesario elaborar más pruebas de caracterización para conocer la interacción entre la superficie de sacrificio y el mortero de nueva incorporación en el sistema constructivo del inmueble histórico, para asegurar la durabilidad y conservación del patrimonio histórico.

La utilización de agregado reciclado en el sector de la construcción tiene diversas ventajas, una de ellas es la disponibilidad del material, al ser residuo de una industria local 
facilitaría su transporte; sin embargo, al no contar un proceso de obtención estándar disponer de un volumen requerido de forma regula para una obra de intervención representaría un reto; no obstante, al ser un material natural, disponible en la atmosfera no genera un impacto su producción.

Los resultados son muy prometedores pues la actividad puzolánica toma su tiempo en desarrollarse y el polvo muestra que aún a edades tempranas se observó su actividad, también se incide en otras áreas del conocimiento como el ciclo de vida, la disminución de los residuos sólidos provenientes de otros procesos; lo relativo a lo estético al lograr que el color sea integral y no una membrana superficial.

Los autores agradecen al Consejo Nacional de Ciencia y Tecnología, por su valioso apoyo para la realización de esta investigación. Al sistema SEP Prodep, a la Coordinación de la Investigación Científica de la UABJO También agradecen al personal del Laboratorio de Materiales "Tecnología y Sustentabilidad" Universidad Autónoma "Benito Juárez" de Oaxaca por el apoyo.

\section{LISTA DE REFERENCIAS}

Álvarez, J. (2005). Caracterización de morteros para revestimiento con polvo de piedra. Revista de la Construcción, 30-37. Disponible en: http://www.redalyc.org/articulo.oa?id=1276/127619745004

Amaral, L., Girondi, G., Nicolite, M., \& Teixeira, M. (2020). Eco-friendly mortars with addition of ornamental stone waste - A mathematical model approach for granulometric optimization. Journal of Cleaner Production. https://doi.org/10.1016/j.jclepro.2019.119283

Arizzi, A., \& Cultrone, G. (2012). Aerial lime-based mortars blended with a pozzolanic additive and different admixtures: A mineralogical, textural and physicalmechanical study. Construction and Building Materials, 135-143. https://doi.org/10.1016/j.conbuildmat.2011.12.069

Betancourt, J., Lizarraga, L., Narayanasamy, R., Olguin, F., \& Sáenz, A. (2015). Revision sobre el uso de residuos de mármol, para elaborar materiales para la construccion. Revista de la Arquitectura e Ingenieria,, 02-12. Disponible en: https://www.uaeh.edu.mx/investigacion/productos/7250/. 
Cedeño, A. (2011). Aglomerantes, morteros y aplanados adecuados para proteger el medio ambiente. Tecnología, medioambiente y sostenibilidad, 106-117. Disponible en: https://www.researchgate.net/publication/237023530.

Chan Yam, J. L., Solís Carcaño, R., \& Moreno, E. I. (2003). Influencia de los agregados pétreos en las caracteristicas del concreto. Ingenieria, 39-46. Disponible en: https://www.virtualpro.co/biblioteca/influencia-de-los-agregados-petreos-en-lascaracteristicas-del-concreto.

Corinaldesi, V., Giuggiolini, M., \& Moriconi, G. (2002). Use of rubble from building demolition in mortar. Waste Management, 893-899. https://doi.org/10.1016/S0956-053X(02)00087-9.

Francisco, A., \& Sanchez, B. (Octubre de 2009). Análisis de la reutilización de residous procedentes de la industria de silestone en la fabricación de mezclas bituminosas. Granada, España. Disponible en: https://dialnet.unirioja.es/servlet/tesis?codigo $=64102$.

Gisbert Aguliar, J., Mateos Royo, I., \& Somovilla de Miguel, I. A. (2015). Morteros de restauración. ResearchGate, 11-21. Disponible en: https://www.academia.edu/18389190/MORTEROS_DE_RESTAURACI\%C3\% $93 \mathrm{~N}$.

International, A. (25 de Mayo de 2018). Método de ensayo normalizado para la determinación granulométrica de agregados finos y gruesos. Método de ensayo normalizado para la determinación granulométrica de agregados finos y gruesos. Washingtong, Estados Unidos: ASTM.

Jiménez, J., Ayuso, J., Fernandez, J., \& de Brito, J. (2013). Use of fine recycled aggregates from ceramic waste in masonry mortar manufacturing. Construction and Building $\quad$ Materials, 679-690. https://doi.org/10.1016/j.conbuildmat.2012.11.036.

Klemm, A. W. (2016). Sustainability of natural stone as a construction material. Woodhead Publishing Series in Civil and Structural Engineering, 283-308. https://doi.org/10.1016/B978-0-08-100370-1.00012-3.

Lanas, J., \& Alvarez, J. (2006). Preparación y ensayos de morteros de cal de nueva factura para su empleo en restauración del patrimonio. V Jornada "Tecnicas de 
restauracion $y$ conservacion del patrimonio, 1-14. http://digital.cic.gba.gob.ar/handle/11746/1492.

Lopes Lima, P. R., \& Batista Leite, M. (2012). Influence of CDW recycled aggregate on drying shrinkage of mortar. Scientific Research, 53-57. DOI:10.4236/ojce.2012.22009.

Luque, A., Sebastian Pardo, E. M., \& de la Torre Lopez, M. J. (2006). Estudio comparado de morteros de cal en pasta y cal en polvo. Control de la carbonatación. MACLA: Revista de la Sociedad Española de Minerología, 293-296. Disponible en: https://dialnet.unirioja.es/servlet/articulo?codigo $=6346781$.

Materials, A. S. (2001). Standard Test Method for Sieve Analysis of Fine and Coarse Aggregates ASTM C136 - 01. West Conshohocken: American Society for Testing and Materials.

Matias, G., Faria, P., \& Torres, I. (2014). Lime mortars with ceramic wastes: Characterization of components and their influence on the mechanical behavior. Construction and Building $\quad$ Materials, 523-534. https://doi.org/10.1016/j.conbuildmat.2014.09.108.

Navarro Sanchez, L. M., Martinez Molina, W., \& Espinoza Mandujano, A. (2011). Analisis de materiales. Morelia, Michoacan: Universidad Michoacana de San Nicolas de Hidalgo.

Normas, D. G. (16 de Noviembre de 2016). Industria de la construcción-cementos hidraulico-determincacion de la resistencia a la compresion de cementantes hidraulicos. Industria de la construcción-cementos hidraulico-determincacion de la resistencia a la compresion de cementantes hidraulicos (CANCELA A LA NMX-C-061-ONNCCE-2001). Ciudad de México, Ciudad de México, México: NMX.

Pavía, S., \& Treacy, E. (2006). A comparative study of the durability and behaviour of fat lime and feebly-hidraulic lime mortars. Materials and structures, 391-398. https://doi.org/10.1617/s11527-005-9033-4.

Quiroga, P. N., \& Fowler, D. W. (2003). The effects of agregates characteristics on the performance of portland concrete. Texas: International center for aggregates research.

Disponible

en: 
http://citeseerx.ist.psu.edu/viewdoc/download?doi=10.1.1.468.6998\&rep=rep1\& type $=$ pdf

Rosell, J., \& Bosch, M. (2018). Hormigones de cal: nuevos "viejos" materiales. VI Jornadas FICAL, 76-86. Disponible en: https://upcommons.upc.edu/bitstream/handle/2117/118912/Fical2018.pdf;jsessio nid $=01026964 \mathrm{C} 284 \mathrm{~A} 82896 \mathrm{FA031F} 8 \mathrm{DF} 21398$ ? sequence $=6$.

Sites, I. C. (2011). Illustred Glosary on Stone Deterioration Patterns. Paris: International Scientific Committee for Stone (ISCS).

Terán Bonilla, J. A. (2004). Consideraciones que deben tenerse en cuenta para la restauración arquitectónica. Conserva, 101-122. Disponible en: https://www.cncr.gob.cl/611/w3-article-4673.html?_noredirect=1.

Veiga, R. (2017). Air lime mortars: What else do we need to know to apply them in conservation and rehabilitation interventions? A review. Construction and Building Materials, 132-140. https://doi.org/10.1016/j.conbuildmat.2017.09.080.

Waste, P., J. Ball, R., \& Paine, K. (2019). Olivine as a reactive aggregate in lime mortars. Construction and Building Materials, 115-126. https://doi.org/10.1016/j.conbuildmat.2018.11.062. 